Cahiers d'études africaines

\title{
Aimé Césaire
}

La chair des mots, une conscience noire du $\mathrm{xx}^{\mathrm{e}}$ siècle

\section{Romuald Fonkoua}

\section{(2) OpenEdition}

\section{Journals}

\section{Édition électronique}

URL : http://journals.openedition.org/etudesafricaines/11722

DOI : 10.4000/etudesafricaines. 11722

ISSN : $1777-5353$

\section{Éditeur}

Éditions de l'EHESS

\section{Édition imprimée}

Date de publication : 20 septembre 2008

Pagination : 399-411

ISBN : 978-2-7132-2184-2

ISSN : 0008-0055

Référence électronique

Romuald Fonkoua, « Aimé Césaire », Cahiers d'études africaines [En ligne], 191 | 2008, mis en ligne le 29 septembre 2008, consulté le 01 mai 2019. URL : http://journals.openedition.org/ etudesafricaines/11722 ; DOI : 10.4000/etudesafricaines.11722

Ce document a été généré automatiquement le 1 mai 2019.

(c) Cahiers d'Études africaines 


\title{
Aimé Césaire
}

\author{
La chair des mots, une conscience noire du $\mathrm{Xx}^{\mathrm{e}}$ siècle
}

\section{Romuald Fonkoua}

1 En accordant à Aimé Césaire, le dimanche 20 avril 2008, des funérailles nationales, plus d'un demi-siècle environ après Colette (1954) et Paul Valéry (1945) et plus de deux siècles après Victor Hugo (1884), la République française a voulu rendre hommage à la dernière grande figure de la Négritude de langue française. Avec Léon-Gontran Damas (1912-1976) - ce résistant dont on a oublié les conditions rocambolesques de l'enterrement - et Léopold Sédar Senghor (1906-2001) - cet académicien oublié par les autorités politiques au moment de l'inhumation -, le député-maire honoraire de Fort-de-France a incarné de façon singulière la prise de conscience de l'être du Noir au monde qui reste sans doute l'une des caractéristiques de la pensée de notre $\mathrm{xx}^{\mathrm{e}}$ siècle.

2 Loin d'être simplement ce "nègre fondamental ", ce "nègre inconsolé » ou encore ce nègre dont "la traversée du siècle est paradoxale", Aimé Césaire est un "nègrecarrefour $»^{1}$ : celui qui ouvre la voie à la fabrique de cette négritude que toutes ces foules de par le monde rassemblées ont (re)découverte. La disparition de Césaire ouvre pour nous aujourd'hui l'ère d'une relecture critique sereine de l'histoire de cette prise de conscience dont on se propose de relever quelques points saillants à travers son œuvre littéraire et son discours politique. De toute évidence, le rapport aux mots est au cœur de cette réflexion. Souvent incompris parce qu'il était en avance sur son temps, ou parce que refusant de se compromettre par peur des compromissions il apparaissait parfois raide, suivant en toute chose ce qui lui semblait juste, Césaire restera pour toujours cet insolent du verbe qui suscite une admiration légitime et méritée - parfois jusqu’à la caricature ${ }^{2}$.

Un noir antillais à l'école de la République.

Le choix des mots

3 Né en 1913 à Basse-Pointe au Nord-Est de la Martinique, Aimé Césaire va suivre un itinéraire personnel qui ressemble à celui de nombreux intellectuels des colonies françaises de son temps. Il a la chance d'appartenir à une famille où l'instruction et l'éducation en français font déjà partie des usages sociaux. Son grand-père, Fernand Césaire, prématurément disparu en 1896 à l'âge de 28 ans, était directeur d'école à SaintPierre, rue de l'Équerre, après avoir suivi des études à l'École Normale des instituteurs de 
Saint-Cloud. Son père, géreur d'habitation après son brevet d'études élémentaires, achèvera sa carrière comme inspecteur des impôts, grâce à sa réussite à un concours administratif. Quant à sa mère, elle possède un certificat d'études - ce qui est assez rare pour l'époque. Sa famille représente, on le voit, le modèle de cette petite bourgeoisie antillaise schoelcheriste de la fin du $\mathrm{XIX}^{\mathrm{e}}$ siècle et du début du siècle dernier qui s'est élevée dans l'échelle sociale grâce aux études et aux concours administratifs.

4 La scolarité de Césaire est à l'image de son éducation, laïque et républicaine. Dans son île natale, il fréquente l'école primaire du Lorrain et réussit brillamment le certificat d'études. Boursier, il poursuit des études secondaires au collège Schoelcher (avant la construction du lycée désormais célèbre) situé face au Palais du Gouverneur - l'actuel Hôtel de la Préfecture - de Fort-de-France, qu'il achève par un baccalauréat de Lettres. Obtenu à l'âge de 18 ans en 1931, ce diplôme lui permet d'échapper à une île où il étouffe déjà, comme il le notera lui-même, à plusieurs reprises, dans ses conversations publiques ou privées ou dans ce célèbre passage de son Cahier d'un retour au pays natal : « Partir. Mon cœur bruissait de générosités emphatiques » $(1987: 22)$.

Outre l'éveil aux émois amoureux, la rencontre avec Suzanne Césaire et les joies de la paternité, la France lui ouvre tour à tour les portes de Paris et de sa proche banlieue, de la khâgne de Louis-le-Grand et de ses élites (de métropole et des colonies), de l'École Normale supérieure de la rue d'Ulm et de l'agrégation - qu'il préparera mal et ratera. En plus de cette formation intellectuelle classique (latin, grec, philosophie), le séjour en France lui permet des rencontres décisives. Celle avec Léopold Sédar Senghor est la plus marquante: celui qui l'accueille comme son bizu - selon une tradition bien ludovicienne - deviendra au fil du temps son " plus que frère », son «Frère aimé », son « ami $»^{3}$ et lui fera découvrir l'Afrique noire. La rencontre avec Petar Guberina est d'une autre envergure. La découverte de l'Europe centrale, de la Yougoslavie en particulier, servira de détonateur à l'explosion créatrice de son premier et célèbre long poème. Les prémices de la Seconde Guerre et de la conflagration de l'Europe, dont on ne dira plus les conséquences sur l'histoire, les sociétés et la pensée occidentales, vont conduire à la métamorphose de la poésie et de la pensée de Césaire.

Dans cette génération de khâgneux et de normaliens de l'Entre-deux-guerres, Aimé Césaire est un être à part. Externe parce que sa bourse n'est pas très élevée, il doit se contenter d'habiter du côté de Charenton et se rendre tous les jours à Louis-le-Grand en empruntant les transports en commun (métro et bus). À la différence d'un Senghor qui va nourrir en partie grâce à son statut d'interne au sein de l'institution académique de solides amitiés, et, en dehors de celles-ci, un réseau de relations tant politiques que littéraires dont on mesurera l'influence tout au long de son itinéraire personnel ${ }^{4}$, Césaire reste comme en dehors du coup, marginal, ou pour reprendre une expression qu'il affectionnera sur la fin de sa vie, un " protestant ».

La négritude : mettre un mot sur l'être noir

7 Le Cahier d'un retour au pays natal, publié dans le $\mathrm{n}^{\circ} 20$ de la revue Volontés, connaît une fortune assez singulière comme la critique l'a déjà plusieurs fois rappelée. Paru deux semaines à peine avant le retour du jeune normalien à la Martinique avec la bénédiction de Pierre Petitbon, professeur à la rue d'Ulm, et de Georges Pellorson, directeur de ladite revue, le poème passe «inaperçu», comme le note Breton. Si les circonstances historiques ne sont pas favorables - nous sommes à la veille de la guerre -, le statut de cette revue n'y aide pas non plus: elle se semble pas avoir contribué de façon extraordinaire à l'avènement de l'art poétique au cours des années 1920-1930 en 
accueillant des figures incontestables de l'intelligentsia française de l'époque qu'aurait pu croiser Breton. Un parcours rapide de ses livraisons antérieures pourrait nous en convaincre aisément. Le poème de Césaire ne connaîtra un certain succès qu'à partir de 1943. André Breton, qui dit l'avoir découvert par les grâces du hasard dans une mercerie tenue par une des sœurs Ménil au cours d'une escale forcée dans l'île durant la guerre ${ }^{5}$, en sera si ébloui au cours de son séjour américain, qu'il va le faire traduire immédiatement en anglais.

Césaire y développe, en partie, la théorie de la négritude qu'il avait déjà conçue en 1935 dès son premier article, "Nègrerie : jeunesse noire et assimilation ", paru dans une revue de portée fort limitée, L'étudiant noir. Il essaie de répondre à "l'admirable question »: «Qui et quels nous sommes?» (1987: 28).

Longtemps, sur ce sujet, la critique a voulu opposer - non sans quelque raison - la négritude «africaine», «ontologique » voire «essentialiste» ou «culturaliste » de Senghor et la négritude " antillaise », " militante » et " de combat ». Chez Césaire, cette autre négritude ne prend corps qu'à partir d'une vision réaliste et dynamique de l'histoire des Noirs du nouveau monde. Il rappelle les conditions de l'esclavage avec son cortège de négations, de souffrances, de soumissions, de dénigrements et de dénis :

«Non nous n'avons jamais été amazones du roi du Dahomey, ni princes du Ghana avec huit cents chameaux, ni docteurs de Tombouctou Askia le Grand étant roi, ni architectes de Djenné, ni Madhis, ni guerriers. Nous ne nous sentons pas sous l'aisselle la démangeaison de ceux qui tinrent jadis la lance. Et puisque j'ai juré de ne rien céler de notre histoire (moi qui n'admire rien tant que le mouton broutant son ombre d'après-midi), je veux avouer que nous fumes de tout temps d'assez piètres laveurs de vaisselle, des cireurs de chaussure sans envergure, mettons les choses au mieux, d'assez consciencieux sorciers et le seul indiscutable record que nous ayons battu est celui d'endurance à la chicotte » $(1987: 38)^{6}$.

10 Ces circonstances de l'esclavage distinguent l'histoire du Nègre des Antilles de l'histoire du Nègre d'Afrique. Elles contribuent aussi à créer son rapport particulier au monde et la qualité particulière de sa négritude :

«Ma négritude n'est pas une pierre, sa surdité ruée contre la clameur du jour/ma négritude n'est pas une taie d'eau morte sur l'œil mort de la terre/ma négritude n'est ni une tour ni une cathédrale. Elle plonge dans la chair rouge du sol/Elle plonge dans la chaleur ardente du ciel/elle troue l'accablement opaque de sa droite patience » $(1987: 46)$.

11 Sa négritude va bien au-delà de l'identité affirmée à partir de l'un - pour parler comme Glissant: un unique territoire, une seule race, une couleur identique ou une culture spécifique. Elle désigne une négritude historique multiple, c'est-à-dire produite par les circonstances de la globalisation et témoignant de tous les aspects de celle-ci. En d'autres termes, la négritude n'est "pas un indice céphalique, ou un plasma, ou un soma, mais mesurée au compas de la souffrance » (1987: 56). Elle ne saurait être qu'à la couleur originale d'une géographie humaine déterritorialisée, « une géométrie du sang répandu » (1987 : 56). Retournant le sens « ontologique » premier dans lequel certains «savants » européens ont figé le mot, Césaire va faire du mouvement et de la dynamique les conditions inhérentes à l'avènement du Nègre dans l'histoire :

«Eia pour ceux qui n'ont jamais rien inventé/mais ils s'abandonnent, saisis, à l'essence de toute chose/ignorants des surfaces mais saisis par le mouvement de toute chose/insoucieux de dompter, mais jouant le jeu du monde/véritablement les fils aînés du monde/poreux à tous les souffles du monde/aire fraternelle de tous les souffles du monde/lit sans drain de toutes les eaux du monde/étincelle du feu sacré 
du monde/chair de la chair du monde palpitant du mouvement/même du monde »

(1987 : 47). préoccupations constantes de Césaire. À travers les sens accordés à la négritude, les discours du Cahier visaient d'abord à redessiner une image nouvelle du nègre au monde. Le poète martiniquais reprenait à son compte dans l'espace sociohistorique français et à travers le cas spécifique de la langue française, le mouvement qui avait conduit les poètes noirs-américains des années 1920 à proclamer l'avènement du New Negro. On ne sera donc pas surpris que le prisme noir américain l'ait poussé à traduire les écrivains de la negro renaissance de Harlem (Jean Toomer, James Weldon Johnson, Claude Mac Kay); à se nourrir de l'expérience ancienne de leur rapport au monde pour tenter de comprendre celle des nègres de l'espace français. inversant ceux qui avaient forgé la négative inversant ceux qui avaient forgé la négative image du noir, mais en retrouvant cette manière primitive de saisir le monde qui n'aurait jamais dû se perdre aux fils du temps et de l'histoire de la civilisation. Optimiste, Césaire estime en effet que « l'œuvre de l'homme n'est point finie ", qu'il n'est pas vrai « que nous n'avons rien à faire au monde » ou « que nous parasitons le monde " ou encore "qu'il suffit que nous nous mettions au pas du monde » (1987 : 57). Il pense au contraire qu'il est temps pour le nègre de se « ceindre les reins comme un vaillant homme» (1987:50), de prendre sa part pleine et entière à l'histoire du monde, ou pour reprendre l'expression qu'il va utiliser dans son discours prononcé à la Sorbonne à l'occasion du $1^{\mathrm{er}}$ Congrès international des écrivains et artistes noirs de 1956, «d'entrer sur la scène de l'histoire du monde » afin de témoigner de «l'œuvre de l'homme».

Réinventer le monde : une politique du mot

Dans un passage célèbre et énigmatique du Cahier, Césaire pensait qu'il fallait commencer par hâter la «fin du monde », car c'était « la seule chose au monde qu'il vaille la peine de commencer " (1987: 32). Mais la destruction du monde ancien est insensée si elle ne s'accompagne d'une gésine nouvelle et d'une parturition. Toute l'œuvre de Césaire va prendre dès lors une dimension politique totale.

Du point de vue de l'organisation (ou de la réorganisation) de la cité - qui est le sens immédiat du politique -, Césaire dénonce les logiques qui ont régi les relations entre les nouveaux et le vieux monde. Aux Antilles mêmes, il s'oppose au système administratif mis en place par les colons à leur profit depuis des siècles et qui fonctionne encore dans des termes identiques au cours de la première moitié $d u x^{e}$ siècle comme l'avaient déjà perçu quelques années plus tôt les rédacteurs de la revue Légitime défense (1932/1). On se souviendra qu'une bourgeoisie noire antillaise, se réclamant du socialisme, avait soutenu, accompagné et parfois encouragé - activement ou par un silence complice - la dépossession économique des îles et l'aliénation culturelle des "coupeurs de canne ». Chez Césaire, l'adhésion explicite au Parti communiste et à ses idéaux est tardive - si on la compare à celle d'un René Ménil par exemple. Mais les vues idéologiques du Parti au lendemain de la guerre, ses prises de positions aux Antilles mêmes et, plus généralement, dans les luttes de libération des peuples opprimés lui étaient apparues comme des arguments valables.

16 C'est au nom de cette lutte pour la liberté totale des peuples qu'il entame, dès 1941, avec un groupe de compagnons, la longue aventure de la revue Tropiques (1941-1945). L'entreprise a valeur de manifeste comme le montre la querelle qui va s'organiser sur ses 
fonts baptismaux. Le chef du service d'information de la Martinique, le Lieutenant de Vaisseau Bayle, qui devait fournir aux rédacteurs de ladite revue - selon les pratiques en vigueur sous Vichy - le papier nécessaire à l'impression du premier numéro avait choisi par provocation ou par inconscience la date du 10 mai pour émettre des objections sur la liberté d'opinion dont pourraient se targuer les auteurs. Il écrivait : « La liberté ? Certes, mais pas la liberté d'empoisonner les esprits, de semer la haine, de ruiner la morale $»^{7}$. Césaire lui répond en justifiant ses positions par celles identiques des écrivains français et américains les plus mémorables:

«"Empoisonneurs d'âmes" comme Racine au dire des Messieurs de Port Royal.

"Ingrats et traîtres à notre si bonne patrie" comme Zola, au dire de la presse réactionnaire.

"Révolutionnaires" comme l'Hugo des « Châtiments ".

"Sectaires" passionnément comme Rimbaud et Lautréamont.

"Racistes" oui. Du racisme de Toussaint Louverture, de Claude Mac Kay et de Langston Hugues - contre celui de Drumont et de Hitler $»^{8}$.

Ce conflit oppose, on le voit, deux conceptions de la pensée française en admettant qu'on puisse utiliser ce mot pour l'une d'entre elles. L'une, révolutionnaire au sens des autorités de Vichy, c'est-à-dire "nationale ", l'autre, révolutionnaire au sens des idéaux du siècle des Lumières, humaniste et universaliste. Il s'articule aussi autour des propriétés du langage. Il met aux prises ceux qui le maîtrisent et en connaissent les usages et les bienfaits, et ceux qui le redoutent justement parce qu'ils reconnaissent sa force vitale. Les auteurs de la revue Tropiques voulaient subvertir par le culturel l'ordre des pouvoirs, religieux (représenté par $\mathrm{M}^{\mathrm{gr}}$ Varin de la Brunelière) et administratif (représenté par l'amiral Robert, gouverneur de la Martinique) pour qui la privation systématique des libertés des nègres aux îles était nécessaire au maintien de la paix civile. Césaire y voyait pour sa part sinon le retour des temps anciens de l'esclavage, du moins les signes de la constante dépossession de l'homme noir sous les tropiques. Selon lui, il fallait créer une nouvelle culture et une nouvelle civilisation, inventer en somme une nouvelle cité humaine. Dans cette minuscule petite terre des Antilles, il fallait donner de la valeur à tout ce qui était considéré comme inexistant ou indigne d'intérêt. Tropiques sera ainsi la première arme de combat public de Césaire durant la guerre, une arme d'autant plus redoutable qu'elle est maintenue par de jeunes hommes - Césaire a 28 ans - pleins de confiance en l'avenir du monde malgré les nuages qui s'amoncellent outre-Rhin, dynamiques et insouciants, confiants dans leur seule capacité à faire œuvre utile. En s'opposant donc à la politique de Vichy dans leur lettre d'intention, les auteurs de la revue Tropiques ne visaient rien moins que l'invention d'une nouvelle manière de penser le monde :

«Terre muette et stérile. C'est de la nôtre que je parle. Et mon ouïe mesure par la Caraïbe l'effrayant silence de l'Homme. Europe. Afrique. Asie. J'entends hurler l'acier, le tam-tam parmi la brousse, le temple prier par les banians. Et je sais que c'est l'homme qui parle. Encore et toujours et j'écoute. Mais c'est l'atrophiement monstrueux de la voix, le séculaire accablement, le prodigieux mutisme. Point de ville. Point d'art. Point de poésie. Point de civilisation, la vraie, je veux dire cette projection de l'homme sur le monde; ce modelage du monde par l'homme; cette frappe de l'univers à l'effigie de l'homme » (Tropiques, 1941/1:5).

Le choix de Césaire est politique si on admet, avec Jacques Rancière, que la politique ne consiste pas seulement à administrer des cités mais à faire coïncider des mondes; à inventer les mots de cette coïncidence ${ }^{9}$. Pour Rancière, la politique est aussi - et très largement depuis l'Antiquité grecque - une affaire de mots. Faire entendre par les mots 
assourdissants la voix assourdie de l'opprimé, telle est donc la seconde exigence politique qui anime Césaire.

Dans le Cahier, on s'en souvient, il avait tenté de donner une « chair aux mots » - selon la belle expression de Jacques Rancière. Les mots n'étaient plus des mots mais des corps. C'étaient des "quartiers de monde ", des "raz-de-marée et des érésipèles", "des paludismes et des laves », « des feux de brousse, des flambées de chair et des flambées de villes... » $(1987$ : 33). À partir de Tropiques, les mots seront ceux du bâtisseur de cathédrale. L'élection - à la mairie de Fort-de-France en mai 1945 puis à l'Assemblée nationale en octobre de la même année - éveillera chez lui la conscience d'une mission à accomplir et d'un devoir à remplir, bref d'un engagement total qui s'exprime sans détour dans les quelques vers suivants des Armes miraculeuses:

«Toute l'eau de Kananga chavire de la Grande Ourse à mes yeux/ [...] mes yeux d'exécution sommaire et de dos au mur/mes yeux qui s'insurgent contre l'édit de grâce/mes yeux de Saint-Pierre bravant les assassins sous la cendre morte/des purs mille défis des roses de Jéricho/ [...] je ne lâcherai pas l'ibis de l'investiture folle de mes mains en flammes » («Investiture », 45).

C'est sur le plan de l'action politique locale que le passage du mot en chair et en corps prendra une part importante de sa valeur. Césaire est en effet un magistrat de la pierre. Il faudra désormais porter à son crédit, l'assainissement de la ville de Fort-de-France resté largement en projet depuis une ordonnance de Colbert à la fin du XVII ${ }^{\mathrm{e}}$ siècle (1681). Cette entreprise posait des problèmes techniques d'autant plus insurmontables que la ville était marécageuse et son premier édile incompétent dans l'aménagement du territoire. Sa réalisation était d'autant plus incertaine que la municipalité manquait de moyens financiers propres et ne possédait aucune garantie viable auprès des bailleurs de fonds. Il faudra encore porter à son crédit la construction d'une ville entière, le célèbre quartier de Trenelle sur les hauteurs de Fort-de-France, qui témoigne aujourd'hui d'une prouesse et d'une totale inconscience financière de son logisticien. Césaire voulait «du bidonville faire une ville et, de la ville elle-même, faire une cité au sens latin du terme - autrement dit une communauté de citoyens libres $»^{10}$. Pour arriver à ses fins, il va faire de la communauté urbaine le premier employeur de l'île :

«Je suis arrivé à réunir ici presque trois mille ouvriers. Il fallait leur donner du travail. Une vraie gageure. Ce n'était pas très raisonnable, mais, enfin [...]. J'ai fait de mon mieux, de manière imprudente, souvent, parce que je ne connaissais pas l'administration [...]. L'eussé-je connue, d'ailleurs, que cela n'aurait pas servi à grand-chose ${ }^{11}$.

21 Bel aveu! Pour Césaire, seule la fin justifiait les moyens. Et ces moyens défiaient les raisons du Trésor public français qui va tenter (et réussir parfois) la mise sous tutelle de sa gestion municipale.

Le mot va se faire chair et corps aussi sur le plan national. Cet autre aspect procède d'abord d'une pratique de pouvoir qui ressemble mutatis mutandis à celle en usage dans la Grèce antique. Césaire a choisi et s'y est largement tenu une forme de démocratie directe. Respectueux de la parole donnée, du lien indéfectible qui doit lier le représentant à son peuple, il a toujours voulu tenir compte de l'opinion de ce dernier, se conformer à ses décisions exclusives, s'aliéner au peuple en somme au risque d'apparaitre aux yeux des autorités administratives de l'île comme un populiste. Cette pratique était rendue nécessaire par les contraintes géographiques et historiques de l'exercice de sa fonction de député. Alors qu'un élu métropolitain pouvait habiter sa circonscription et garder le contact étroit avec les populations, Césaire, lui, ne pouvait se rendre qu'irrégulièrement 
dans son île. Son retour donnait souvent lieu à des meetings où il faisait un compte rendu exhaustif de son activité durant la session écoulée et attendait les doléances des populations ${ }^{12}$. En outre, dans un monde où l'information ne circulait encore qu'avec retard (lorsque la presse était plus de propagande que d'opinion), la parole de l'élu était la seule autorité crédible pour les populations et, en retour, la voix réelle de ces dernières. Cette communication politique est aussi une osmose entre l'élu et son peuple. Tous deux ne forment plus désormais qu'un même corps. La communion va pousser Césaire à réclamer et à obtenir au lendemain de l'adoption de la Constitution de 1946 le statut de Département pour la Martinique et trois autres îles anciennes (Guadeloupe, Guyane, Réunion). Bien qu'il ait toujours été assez lucide sur l'opportunité de cette mission, il obtenait néanmoins dans la seconde moitié $d u x^{e}$ siècle ce que les populations des vieilles colonies espéraient depuis plus de deux cents ans, c'est-à-dire depuis l'abolition de l'esclavage de 1848 et l'avènement de la liberté politique : l'égalité parfaite, pleine et entière des citoyens des îles dans la République française. Au demeurant, cette victoire achevait de fort belle manière l'œuvre du Libérateur, Schoelcher, à qui le poète avait rendu un hommage appuyé dans la revue Tropiques (1945/13-14: 227-243) à travers des propos élogieux qui contrastaient avec l'image sinon hautaine du moins neutre qui se dégageait du Cahier.

Toutefois, donner de la chair aux mots ne fut pas toujours à la hauteur du pari. Le terme "égalité " prenait les formes de l'«assimilation »: celle que le poète redoutait dès son premier article, « Nègrerie : jeunesse noire et assimilation », évoqué plus haut, revenait sur la scène publique. Alors que le poète, en politique, espérait que cette égalité de droit imposerait une égalité de fait, que les Antilles franchiraient rapidement l'obstacle des lois qui les séparaient de la justice sociale, des allocations familiales et des congés payés en vigueur dans la France métropolitaine, les gouvernements successifs lui opposaient une logique comptable. Au grand rêve de la démocratie sociale du poète s'opposait une fois de plus la grande réalité boutiquière. Chassée par la force du langage poétique qui lui avait dénié tout droit de cité, l'assimilation se réintroduisait par la porte imprévue de l'accession à la citoyenneté. La politique avait signifié jusque-là pour lui un accord sur et avec les mots. Elle va désigner très vite, en plus des contraintes liées à sa manifestation naturelle (l'organisation de stratégies, la mise en place et l'usage d'une rhétorique), un éternel combat pour le sens réel du mot adéquat.

La qualité du verbe ou le sens réel du mot adéquat

Les batailles menées par Césaire au Palais Bourbon au cours des premières années de la départementalisation vont lui faire prendre conscience des contraintes du jeu parlementaire et de la vanité des mots. Dès le début des années 1950 commence pour lui une longue période de doute qui va être ponctuée par d'essentielles ruptures. Hier encore, il pensait que grâce à son mystère, le mot était une arme miraculeuse. Il constate au contraire que le miracle du mot n'est rien si ce dernier n'est accompagné de structures de contrôle qui rendent sa compréhension intangible. Plus exactement, c'est en contrôlant l'organe de diffusion du message qu'on contrôlera le message lui-même; et c'est en forgeant pour le Nègre une idée à l'aune de son être qu'on contribuera à donner un vari corps aux « mots de la tribu ».

L'occasion va lui être donnée dès la fin de l'année 1955 avec la préparation et la réussite $\mathrm{du} 1^{\mathrm{er}}$ Congrès des écrivains et artistes noirs de la Sorbonne du 19 au 22 septembre 1956. En plus de sa participation active à l'organisation de ces premières rencontres culturelles du monde noir, Césaire choisit de faire des éditions Présence Africaine créées en 1947 par 
Alioune Diop (1910-1980) sa maison: elle saurait mieux que toutes les autres faire entendre sa voix sans la censurer et ses mots sans les déformer ou les trahir. Il y publie notamment la version définitive du Cahier, puis l'adaptation théâtrale de Et les chiens se taisaient. Il y réédite également, dès 1955 , son fameux Discours sur le colonialisme ${ }^{13}$ ainsi que la célèbre « Lettre à Maurice Thorez » qui signe sa démission du PCF. Ses œuvres majeures, c'est-à-dire sa parole essentielle, seront ainsi placées à l'abri de tout type de persécution - comme la mise au silence progressive à laquelle il a été contraint durant l'exercice du mandat de député. Poursuivant cet engagement, il accepte de présider jusqu'en 2003, après Jean Price-Mars (1876-1969), la Société africaine de culture (SAC), une organisation internationale de culture non gouvernementale.

La confiance qu'il accorde à la maison d'édition et le soutien indéfectible dont il bénéficie de son directeur, Alioune Diop, assurent le poids d'une autre qualité que vont prendre désormais ses mots. Alioune Diop avait conçu l'idée d'une maison d'édition qui serait l'espace authentique de l'expression d'une pensée noire autonome. Césaire lui emboîte le pas dans sa lettre de démission du PCF en affirmant que "l'heure de nous mêmes a sonné ». Ayant acquis la conviction, au regard de l'histoire récente du communisme et particulièrement des méfaits du stalinisme dénoncés par le rapport Kroutchev, que les voies du marxisme et de l'anticolonialisme ne se confondaient pas nécessairement, il estime « qu'aucune doctrine ne vaut que repensée par nous, que repensée pour nous, que convertie à nous. Cela a l'air d'aller de soi. Et pourtant dans les faits cela ne va pas de soi ».

28 En effet, si le communisme avait permis de comprendre le rapport des exploités à leur monde d'un point de vue général, le tort des communistes staliniens avait été d'accepter que des inégalités historiques et raciales aient quelque place dans une pensée de l'égalité ; de faire de la subordination des peuples un des modes d'organisation politique. De plus, les nombreuses singularités antillaises : celle de «notre situation dans le monde»; de « nos problèmes qui ne se ramènent à nul autre problème »; de " notre histoire coupée de terribles avatars qui n'appartiennent qu'à elle »; de " notre culture que nous voulons vivre de manière de plus en plus réelle ", entraînaient nécessairement vers d'autres voies que « celles qui ont mené à l'imposture, à la tyrannie, au crime ». Pour Césaire, le Nègre ne devait plus se contenter «d'assister à la politique des autres. Au piétinement des autres. Aux combinaisons des autres. Aux rafistolages de consciences ou à la casuistique des autres ", mais s'efforcer de créer un discours en adéquation avec son moi.

Si on laisse de côté - bien qu'elle soit importante - l'histoire de la création du Parti progressiste martiniquais (PPM) qui signe concrètement ladite autonomie, on notera que celle-ci s'accompagne de nombreux écrits et essais où Césaire tente de donner un corps théorique aux mots de la doctrine.

Une première réflexion est consacrée à l'histoire avec la publication de son Toussaint Louverture. La Révolution et le problème colonial en 1962. Dans cet important essai - malheureusement trop peu connu -, Césaire dessine une double image antillaise de la révolution. D'une part, contrairement à la lecture centrifuge qui en a toujours été faite (de la métropole vers les îles à la manière de l'histoire de quelques républiques sœurs ${ }^{14}$ ), il montre que les Antilles sont au cœur de la révolution. Ce sont elles qui la justifient et lui donnent sens. D'autre part, contrairement à l'absence remarquable des Antillais dans l'histoire officielle de la Révolution, Césaire estime que Toussaint est sa seule et unique figure réelle: celui qui ne transige pas avec les principes - à la différence de ses homologues français. Chez ce «jacobin noir », la réalisation des idéaux de la révolution 
est d'autant plus nécessaire que l'expérience de l'esclavage a conduit à une déshumanisation totale. Si on suit Césaire jusqu'au bout de sa logique, on peut penser que ce sont les Antilles qui ont su mieux que les autres mettre en musique l'air révolutionnaire sans les mauvais accords que la France métropolitaine n'a pas su éviter dans son histoire. Ici, l'universalisme révolutionnaire pleinement justifié se montre dans toute sa splendeur.

31 La réflexion sur la pratique concrète du pouvoir politique constitue un second aspect. Césaire se veut, en même temps, praticien du politique et observateur impartial de la pratique des politiques des sociétés postcoloniales. Il constate que l'aventure de tout héros révolutionnaire dans les sociétés issues des esclavages, des colonisations et donc des formes de domination est tragique, comprise entre des idéaux qu'il faut atteindre et la réalité concrète qu'il faut assumer; entre les lumières de la libération et ses exigences. Les figures du roi Christophe (Haïti), de Lumumba (Congo) et dans une moindre mesure de Caliban (États-Unis) vont nourrir sa réflexion critique. Autant la figure de Toussaint Louverture est immaculée, autant celle de ces héros révolutionnaires est controversée. Des Amériques noires au Congo en passant par Haïti, Césaire semble avoir été hanté par les dérives dictatoriales qui ont nui gravement à la réalisation des idéaux révolutionnaires. Contraints comme le roi Christophe de «demander aux nègres plus qu'aux autres : plus de travail, plus de foi, plus d'enthousiasme $»^{15}$, ces héros ne peuvent connaître qu'un destin tragique et leur vie une « issue fatale ».

Le théâtre, la mise en scène, l'exposition des dérives des dictatures sont un retour d'investissement sur le réel. L'art préserve l'idéal révolutionnaire dans sa pureté tout comme il prémunit Césaire de ces tentatives toujours grandes de népotisme dont il n'est pas besoin de refaire ici l'histoire récente ou ancienne en postcolonie. Elle lui permet en tout cas de résoudre cet embarras d'avoir à choisir entre la thèse assimilationniste (pertinente pour certains) et la thèse autonomiste (adoptée par d'autres). Pour lui, l'une et l'autre thèse présentaient ce qu'il appelait dans son discours du 22 mars 1958 devant le Congrès constitutif du Parti progressiste martiniquais des "contrariétés ». Comment se dégager du poids des décisions parisiennes qui sont bien souvent des diktats imposés de l'extérieur à l'intérieur? Comment imposer des vues autonomes lorsque celles-ci ne sont pas exemptes de dérives autocratiques? Voilà la double postulation contraire que le poète ne peut résoudre qu'au moyen de la tragédie. Il n'est d'ailleurs pas certain que les conditions actuelles de la décentralisation aux Antilles ne renforcent cette dimension tragique du faire politique.

33 En définitive, l'héritage intellectuel, littéraire et politique de Césaire est double : l'appel à la révolution et l'exercice d'une vigilance constante face aux pratiques politiques. Sa lucidité face au pouvoir lui a toujours permis de se méfier de ses jeux sociaux. De fait, il a su garder cette distance nécessaire à la gestion de la chose publique et indispensable à la critique permanente de celle-ci. Mieux que les autres écrivains de la Négritude chez qui le poétique et le politique se confondent, il aura su garder cette conscience première de la vanité des choses qui, au bout du compte, l'a préservé de toute grande compromission ou de toute dérive à la manière du Rebelle de Et les chiens se taisaient. Il n'a pas eu les mêmes faiblesses qu'un Senghor (usant parfois de son pouvoir du mot pour contraindre ses adversaires politiques au silence). Il n'a pas connu ces égarements dus à l'insouciance, la négligence ou à la facilité d'un Damas (oubliant le sens universel des mots pour embrasser une idéologie pacifiste parfois nuisible). Tout à la fois sérieux et distant, il a pratiqué l'art de la politique dans le but de réinventer des mondes, et l'art de la littérature avec 
l'intention de ne donner aux mots que des corps de «pur-sang " passionnés qui, « l'oreille collée au sol », entendront toujours « passer demain $»^{16}$.

\section{NOTES}

1. Sur le sens de ce mot appliqué à Césaire, voir Roger ToumSON \& Simone HENRY-VALMORE , Aimé Césaire le nègre inconsolé, Paris, Fort-de-France, Syros (« Vent des îles »), 1993.

2. Voir par exemple le dernier opus de Claude RIBBE, Le nègre vous emmerde, Paris, BuchetChastel, 2008.

3. «Lettre à un poète » (Chants d'ombre, O. P. : 1945, 11). C'est par ces premiers mots que Senghor adresse à Césaire son salut « abrupt et fraternel ». Dans une lettre en notre possession datée du mois d'octobre 2000, Césaire adressait encore à Senghor des vœux d'anniversaire pour ses 94 ans. Jusqu'à son dernier souffle, il n'a pas manqué de s'inquiéter, même de loin, de l'état de santé de Mme Senghor, prenant assez souvent de ses nouvelles par l'intermédiaire de sa fidèle secrétaire.

4. Sur cet itinéraire lire notre article «L'Afrique en Khâgne » repris dans Pierre BRUNEL (ed.), Léopold Sédar Senghor, Poésie complète, Paris, Planète libre CNRS Éditions, ITEM, 2007.

5. Voir. A. BRETON, « Un grand poète noir », préface à l'édition de 1947 reprise en postface de l'édition définitive du Cahier, Paris, Présence Africaine, 1987, p. 79.

6. Cahier d'un retour au pays natal, édition définitive, Paris, Présence Africaine, 1987 [1956].

7. Tropiques, Lettre du Lieutenant du Vaisseau Bayle, chef du service d'information au directeur de la revue Tropiques, « Documents-Annexes », Fort-de-France, le 10 mai 1943, rééd., Paris, J. M. Place, 1978.

8. Réponse de Tropiques, Fort-de-France, 12 mai 1943. Les mots en gras sont repris du texte original.

9. Jacques RANCIÈRE, Aux bords du politique, Paris, La Fabrique, 1998.

10. Patrice LouIs, Conversation avec Aimé Césaire, Paris, Arléa, 2007, p. 51.

11. Patrice LouIs, ibid., p. 52.

12. Le journal Justice, organe d'expression du Parti communiste martiniquais, se fera l'écho régulier de ces diverses prises de parole dans la cour de l'ancienne mairie de Fortde-France, rue Victor Sévère.

13. Discours sur le colonialisme, Paris, Éditions Réclame, 1950.

14. Voir Jean-Louis HAROUEL, Les républiques sœurs, Paris, PUF, 1997 ; Michel VovelLE, Les républiques sœurs sous le regard de la grande nation 1795-1803 : de l'Italie aux portes de l'Empire ottoman, l'impact du modèle républicain français, Paris-Montréal (Québec)-Budapest, L'Harmattan, 2000.

15. La tragédie du roi Christophe, Acte I, sc. 7.

16. « Les “pur-sang” », in Les Armes miraculeuses, Paris, Gallimard (« Poésie, 59 »), 1970

[1946]. 


\section{AUTEUR}

\section{ROMUALD FONKOUA}

Institut de littérature française, Université de Strasbourg. 